\title{
Caracterização da prática de automedicação e fatores associados entre universitários do curso de Enfermagem
}

Characteristics of self-medication practice and associated factors among nursing undergraduate students

Caracterización de la práctica de automedicación y factores asociados entre universitarios del curso de Enfermería

Flávio Martinez da Silva ${ }^{1}$, Flávia Cristina Goulart ${ }^{2}$, Carlos Alberto Lazarini ${ }^{3}$

${ }^{1}$ Enfermeiro. Professor do Centro Universitário Uninter. Curitiba, PR, Brasil. E-mail: flavio_martinez86@hotmail.com.

2 Farmacêutica, Doutora em Neurociências e Comportamento. Professora Doutora da Faculdade de Saúde Pública da Universidade de São Paulo. São Paulo,

SP, Brasil. E-mail: flaviagoulart@usp.br.

${ }^{3}$ Farmacêutico, Doutor em Farmacologia. Professor Titular da Faculdade de Medicina de Marília. Marília, SP, Brasil. E-mail: carlos.lazarini@gmail.com.

\section{RESUMO}

O objetivo do trabalho foi caracterizar a prática de automedicação e os fatores associados entre estudantes ingressantes e concluintes do curso de Graduação em Enfermagem. Utilizou-se estudo quali-quantitativo com questionário semiestruturado aplicado a todos os estudantes da $1^{\text {a }}$ e $4^{\text {a }}$ séries do curso. Os dados quantitativos foram analisados utilizando-se a estatística descritiva e os qualitativos, utilizando-se o Discurso do Sujeito Coletivo. Os resultados mostraram que a maioria dos estudantes da primeira e todos os da quarta série se automedicam. Mostraram, ainda, diferença significativa para faixa etária e consumo de anti-inflamatório entre as séries. Os motivos que levaram os estudantes a se automedicarem foram: influência de terceiros, alívio da dor, demora no atendimento, se perceberem com conhecimentos científicos suficientes após cursarem a disciplina de Farmacologia. Conclui-se que a automedicação é prática frequente entre os estudantes de ambas as séries. Os concluintes, após cursarem Farmacologia, adquirem empoderamento científico suficiente para tal prática.

Descritores: Automedicação; Enfermagem; Estudantes de Enfermagem.

\section{ABSTRACT}

The objective of this study was to characterize self-medication practice and associated factors among freshmen and senior nursing undergraduate students. Our approach was quali-quantitative and we gave a semi-structured questionnaire to all freshmen and senior undergraduate students. Quantitative data were analyzed using descriptive statistics and qualitative data, using the Discourse of the Collective Subject method. The results demonstrate that most first-year and all fourth-year students self-medicate. Furthermore, there were significant differences according to age group and the use of anti-inflammatory drugs between classes. The reasons that led students to self-medicate were: influence of others, pain relief, long waiting periods for receiving care, considering themselves in possession of enough scientific knowledge after taking a Pharmacology class. We concluded that self-medication is a common practice among students from both classes. After taking Pharmacology, seniors feel scientifically empowered enough for this practice.

Descriptors: Self Medication; Nursing; Students, Nursing.

\section{RESUMEN}

Se objetivó caracterizar la práctica de automedicación y factores asociados entre estudiantes ingresantes y concluyentes del Curso de Graduación en Enfermería. Se utilizó estudio cuali-cuantitativo, con cuestionario semiestructurado aplicado a todos los estudiantes de $1^{\circ}$ y $4^{\circ}$ año del curso. Los datos cuantitativos fueron analizados utilizándose estadística descriptiva; los cualitativos, usando el Discurso del Sujeto Colectivo. Los resultados mostraron que la mayoría de los estudiantes de primero y todos los de cuarto año se automedican. También expresaron diferencias significativas para faja etaria y consumo de antiinflamatorios entre los años. Los motivos para la automedicación de los estudiantes fueron: influencia de terceros, alivio del dolor, demora en la atención, percepción de conocimientos científicos suficientes luego de cursar la materia Farmacología. Se concluye en que la automedicación es práctica frecuente entre estudiantes de ambos años. Los concluyentes, luego de cursar Farmacología, adquieren empoderamiento científico suficiente para tal práctica.

Descriptores: Automedicación; Enfermería; Estudiantes de Enfermería. 


\section{INTRODUÇÃO}

A automedicação é entendida como a prática de ingerir medicamentos sem o aconselhamento e/ou acompanhamento de um profissional de saúde qualificado. Em outras palavras, é a ingestão de medicamentos por conta e risco próprios ${ }^{(1)}$. Independente do nível cultural, do contexto histórico envolvido, da posição econômica ou social do indivíduo, a automedicação é uma prática comum, que visa o alívio ou a cura de sintomas considerados simples e recorrentes ${ }^{(2)}$.

Essa prática, mesmo sendo considerada por especialistas como forma comum de autocuidado, pode ser potencialmente danosa à saúde tanto individual quanto coletiva, principalmente pelo fato de que nenhum medicamento é inócuo ao organismo ${ }^{(3)}$. Em farmacologia é sempre preciso analisar homeostaticamente o custo/benefício do uso de cada medicamento e a possibilidade de respostas iatrogênicas. Para tanto, é necessário conhecimentos em farmacologia, bioquímica, fisiologia, patologia, microbiologia e conhecimentos mínimos de interação medicamentosa.

O uso inapropriado de medicamentos e suas causas envolvem uma rede complexa de fatores, dentro e fora da área da saúde. A epistemologia da automedicação em nossa sociedade advém da pajelança e uso de ervas medicinais para tratamentos de doenças da cultura indígena e da cultura popular de curandeirismo usada pelos escravos negros desde os tempos do BrasilColônia. Os escravos tiveram importância significativa no Brasil em relação à história da doença e da cura ao longo do século XIX. Pode-se afirmar, inclusive, que as práticas de cura em nosso país, ao longo de seus primeiros quatro séculos de história foram, antes de tudo, resultantes da troca das experiências terapêuticas entre europeus, índios e africanos. Na sociedade escravocrata os donos dos escravos usavam dos conhecimentos desses para seus tratamentos, como: curas, benzeduras ou o uso das ervas medicinais ${ }^{(4)}$.

Essa cultura da automedicação lesa, porém, de forma principal, o que talvez seja o elo mais frágil desta cadeia: o usuário(5). No Brasil, segundo a Associação Brasileira das Indústrias Farmacêuticas (ABIFARMA), todo ano, cerca de 20.000 pessoas morrem, no país, vítimas da automedicação(6).

Além dos erros de medicação, em termos de políticas públicas, é um desafio reduzir a automedicação em um país que ocupa o sexto lugar entre os países consumidores de medicamentos ${ }^{(7)}$, dispondo de mais de 32.000 medicamentos no mercado, segundo dados da
Organização Mundial de Saúde (OMS) e do Ministério da Saúde.

$\mathrm{Na}$ perspectiva de buscar uma sistematização da prescrição e acesso ao medicamento, o governo federal lançou a Portaria no 3.916/98, do Ministério da Saúde, que estabelece a Política Nacional de Medicamentos, que tem como propósito garantir a necessária segurança, eficácia e qualidade dos medicamentos, a promoção do uso racional e o acesso da população àqueles considerados essenciais $^{(8)}$. Apesar de essa portaria trazer significativas contribuições na discussão dos paradigmas relacionados à compra, distribuição e prescrição de medicamentos pelos órgãos públicos, pode-se verificar que ainda pouco se conseguiu na mudança do hábito de automedicação da população(9).

Para minimizar esse problema, o esperado seria a utilização de medicamentos após indicação de profissional de saúde qualificado(10): médico, dentista ou farmacêutico. Porém, tal qualificação profissional pode ser questionada, uma vez que dados da literatura mostram que acadêmicos da área da saúde possuem o hábito de se automedicar ${ }^{(10)}$. Os percentuais para essa prática podem variar entre as diferentes áreas de formação. Observa-se que $88,57 \%$ dos acadêmicos do curso de enfermagem e $94,55 \%$ dos alunos de medicina praticam esse hábito ${ }^{(11)}$. Além dessas áreas, a automedicação também é encontrada entre estudantes de farmácia, fisioterapia, educação física, nutrição, fonoaudiologia, terapia ocupacional e odontologia(10).

Entre os motivos que levam estudantes universitários a se automedicarem incluem a influência da propaganda; o uso de prescrições antigas; a orientação de funcionários de farmácia, amigos, vizinhos e familiares; o armazenamento de medicamentos em casa; a influência de conhecimento próprio(12) e a cultura do autocuidado com a saúde estabelecida no país. Especificamente para estudantes do curso de enfermagem, observa-se que um dos principais motivos para a realização dessa prática é a presença de dor $^{(13)}$.

Uma vez que o profissional de enfermagem é responsável pela orientação de seus pacientes e pela observância do Protocolo de Segurança na Prescrição, Uso e Administração de Medicamentos faz-se necessário avaliar a prática de automedicação entre estudantes de enfermagem, antes e após a aquisição de conhecimento específico na disciplina de Farmacologia, entendendo que os resultados poderão sustentar propostas para reflexões sobre os paradigmas que norteiam a elaboração dos conteúdos programáticos dessa disciplina e a construção do saber ao que tange o uso racional de medicamentos. 
Sendo assim, o presente estudo teve por objetivo caracterizar a prática de automedicação e os fatores associados entre estudantes ingressantes e concluintes do curso de Graduação em Enfermagem.

\section{MÉTODO}

Estudo descritivo transversal, com abordagem qualiquantitativa realizado em uma instituição pública de ensino superior de um município localizado na região centro-oeste do Estado de São Paulo.

A população em estudo foi composta por todos os estudantes devidamente matriculados na primeira e na quarta séries do curso de Enfermagem da instituição, totalizando 37 e 41, respectivamente. Essas duas séries foram escolhidas para que se possa comparar a prática de automedicação entre os ingressantes e os concluintes e avaliar o quanto a construção de conhecimento na área da saúde ao longo de sua formação pode interferir na mesma. Para tal, foram realizadas entrevistas semiestruturadas, com a utilização de um guia de entrevistas com questões dirigidas para caracterização sociodemográfica, da prática de automedicação e do uso de medicamentos (dados quantitativos) e, uma questão aberta, destinada a conhecer atitudes e comportamentos relativos à prática da automedicação (dados qualitativos).

A coleta de dados foi realizada no primeiro semestre de 2011. Os dados foram coletados por meio de um instrumento único, validado em estudo piloto e padronizado pelo investigador.

Os dados quantitativos foram processados e analisados pelo pesquisador, com a utilização do Software SPSS, versão 17.0. Foram obtidas as frequências absolutas e relativas, e comparadas às variáveis selecionadas no estudo para verificação da existência de associação, utilizando-se o Teste do Quiquadrado ou o Teste Exato de Fisher. Foram consideradas estatisticamente significativas as relações entre variáveis, em que $\mathrm{p} \leq 0,05$.

Apenas os entrevistados que responderam "sim" para a prática de automedicação responderam a pergunta "Por qual motivo você tomou medicamentos sem receita médica?". A resposta a essa pergunta foi gravada em aparelho de MP3, com posterior transcrição e análise. Para o tratamento dos dados qualitativos foi empregada a técnica de análise do discurso do sujeito coletivo $^{(14)}$ que consiste num conjunto de procedimentos de tabulação e organização de dados discursivos, sobretudo (mas não exclusivamente) daqueles provenientes de depoimentos orais. Esses procedimentos envolvem, basicamente, as seguintes operações sobre os discursos coletados:

$\checkmark$ Seleção das expressões-chave de cada discurso particular, por exemplo, de cada resposta a uma questão. As expressões-chave são segmentos contínuos ou descontínuos de discurso que revelam o principal do conteúdo discursivo; são uma espécie de "prova discursivo-empírica" da "verdade" das ideias centrais.

$\checkmark$ Identificação da ideia central de cada uma dessas expressões-chave e que é a síntese do conteúdo dessas expressões, ou seja, o que elas querem efetivamente dizer.

$\checkmark \quad$ Identificação das ideias centrais semelhantes ou complementares.

$\checkmark$ Reunião das expressões-chave referentes às ideias centrais semelhantes ou complementares, em um discurso síntese que é o discurso do sujeito coletivo.

O discurso do sujeito coletivo representa, portanto, um expediente ou recurso metodológico destinado a tornar mais claras e expressivas as representações sociais, permitindo que um determinado grupo social (no caso de estudantes da primeira e da quarta séries do curso de Enfermagem) possa ser visto como autor e emissor de discursos comuns compartilhando entre seus membros.

Este estudo foi aprovado pelo Comitê de Ética em Pesquisa para Seres Humanos da Faculdade de Medicina de Marília, Marília - SP, processo no 396/10, e se iniciou após os participantes assinarem o Termo de Consentimento Livre e Esclarecido.

\section{RESULTADOS}

Todos os estudantes de ambas as séries aceitaram participar da pesquisa. A Tabela 1 mostra os dados referentes à caracterização dos entrevistados. A faixa etária prevalente na primeira série foi de 20 anos ou menos, mostrando existir uma associação estatisticamente significativa entre esta faixa etária e a série. O mesmo pode ser observado na quarta série para a faixa etária entre 21 e 25 anos. Observa-se que na primeira série $86,5 \%$ dos estudantes praticaram a automedicação, e na quarta série esse valor correspondeu a 100\%. A comparação entre as séries mostra existir uma associação estatisticamente significativa para este parâmetro sugerindo prevalência na quarta série. 
Tabela 1: Caracterização dos estudantes regularmente matriculados na primeira e na quarta séries do curso de Enfermagem, Faculdade de Medicina de Marília. Marília, SP, Brasil, 2011.

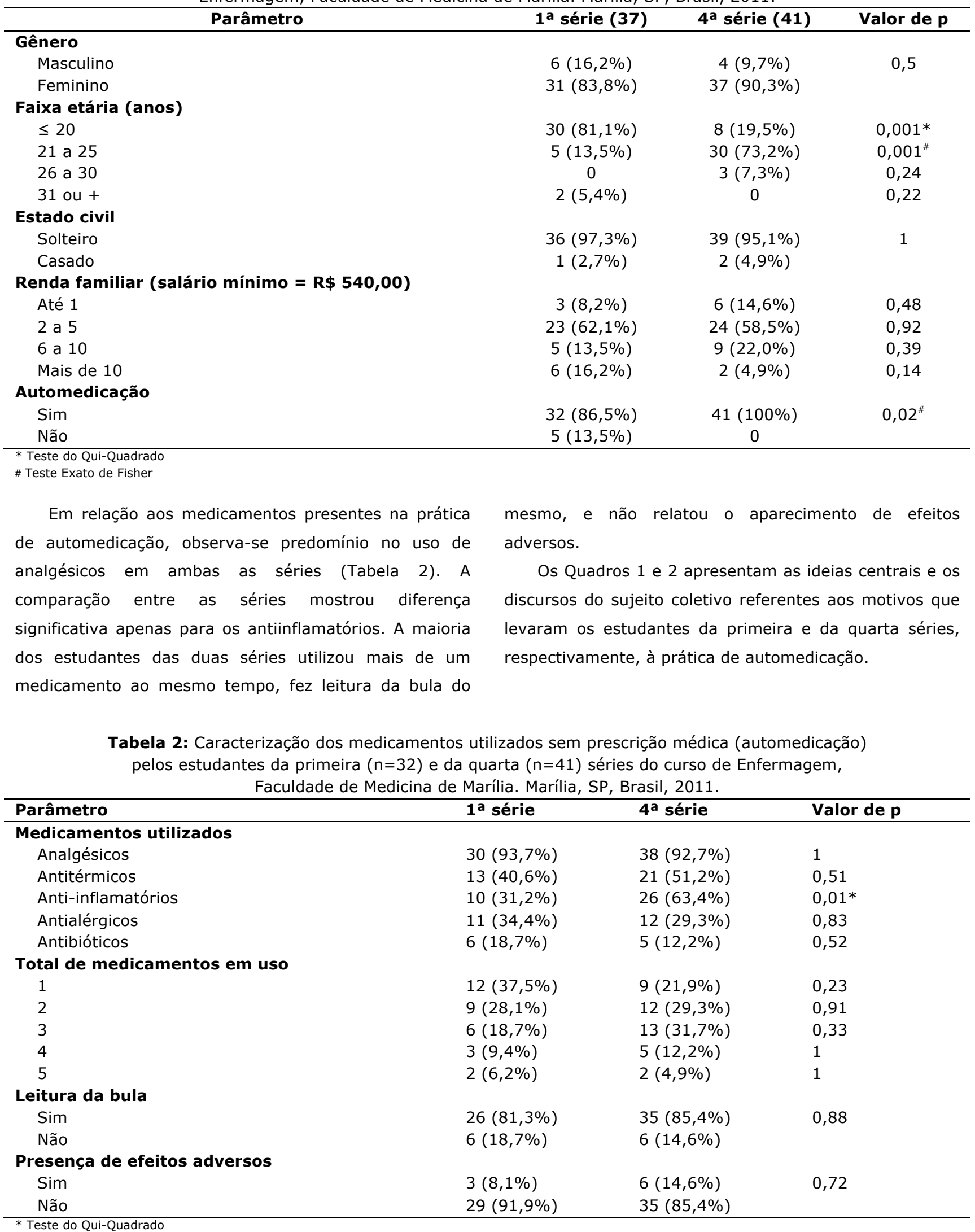


Quadro 1: Ideias centrais e Discursos do Sujeito Coletivo dos 32 estudantes da $1^{\text {a }}$ série do curso de Enfermagem que relataram praticar automedicação. Marília, SP, Brasil, 2011.

\begin{tabular}{|c|c|}
\hline Ideias centrais & Discurso do Sujeito Coletivo \\
\hline $\begin{array}{c}\text { Pela indicação de } \\
\text { terceiros }\end{array}$ & $\begin{array}{l}\text { Sempre fui medicado pelos meus pais, sendo eles instruídos por médicos familiares. Tomo } \\
\text { remédio pra dor de cabeça porque minha mãe me dá, pois confio nos meus pais. O farmacêutico } \\
\text { também nos dá o medicamento necessário. Além disso, tenho profissionais da área da saúde } \\
\text { como amigos... eles me indicam alguns medicamentos. }\end{array}$ \\
\hline $\begin{array}{l}\text { Pela falta de } \\
\text { tempo }\end{array}$ & $\begin{array}{l}\text { Muitas vezes usei medicamentos sem prescrição médica por falta de tempo de ir até uma unidade } \\
\text { de saúde. Por falta de tempo, não vou ao médico, a uma unidade de saúde. Falta tempo de ir ao } \\
\text { médico para pegar uma prescrição. }\end{array}$ \\
\hline $\begin{array}{l}\text { Ter o mesmo } \\
\text { problema }\end{array}$ & $\begin{array}{l}\text { Por conhecer os sinais e sintomas da doença, faço uso dos medicamentos sem prescrição médica, } \\
\text { pois são sintomas iguais todas as vezes que tenho essa doença. Porque achei desnecessário, pois } \\
\text { já havia ido outras vezes e ele (o médico) sempre receita o mesmo medicamento que foi eficiente } \\
\text { antes. }\end{array}$ \\
\hline $\begin{array}{c}\text { Para o alívio da } \\
\text { dor }\end{array}$ & $\begin{array}{l}\text { Não tem necessidade de ir ao médico por doenças pequenas. Não vejo necessidade em usar } \\
\text { prescrição médica com analgésicos, para cessar a dor. Por sentir dores que geralmente são } \\
\text { normais e não precisam de orientação médica. }\end{array}$ \\
\hline $\begin{array}{l}\text { Pela demora no } \\
\text { agendamento }\end{array}$ & $\begin{array}{l}\text { Não vou ao médico pela falta de agilidade do serviço de saúde. Se precisasse esperar uma } \\
\text { consulta médica para pegar a prescrição demoraria muito. }\end{array}$ \\
\hline
\end{tabular}

Quadro 2: Ideias centrais e Discursos do Sujeito Coletivo dos 41 estudantes da $4^{a}$ série do curso de Enfermagem que relataram praticar automedicação. Marília, SP, Brasil, 2011.

\begin{tabular}{|c|c|c|}
\hline $\begin{array}{c}\text { Ideias centrais } \\
\text { conhesuir } \\
\text { científicos }\end{array}$ & $\begin{array}{c}\text { Não senti a necessidade de utilizar o serviço de saúde porque como tenho noção das classes dos } \\
\text { medicamentos e conhecimento quanto à farmacocinética e farmacodinâmica do medicamento a } \\
\text { ser utilizado acabo por entender qual medicamento vai ser o indicado para acabar com } \\
\text { determinado sintoma que estou sentindo no momento. Pois, através do estudo durante a } \\
\text { graduação, adquiri conhecimentos que me dão segurança para me automedicar. }\end{array}$ \\
\hline $\begin{array}{c}\text { Pela facilidade de } \\
\text { acesso }\end{array}$ & $\begin{array}{r}\text { O medicamento estava disponível em casa e a facilidade de compra do medicamento nas } \\
\text { farmácias sem receita médica me dá facilidade ao acesso do medicamento. }\end{array}$ \\
\hline $\begin{array}{c}\text { Para economizar } \\
\text { tempo }\end{array}$ & $\begin{array}{r}\text { Por ser um meio mais rápido de se medicar, isto me permite economia de tempo por não } \\
\text { precisar passar por uma consulta médica. Procurar atendimento médico demanda tempo. O } \\
\text { transtorno de passar em consulta é muito grande... a demora é grande. }\end{array}$ \\
\hline $\begin{array}{c}\text { Utilizar } \\
\text { diagnósticos } \\
\text { prévios }\end{array}$ & $\begin{array}{c}\text { Porque os medicamentos que utilizo sem prescrição médica já foram prescritos para mim para os } \\
\text { mesmos problemas de saúde. São os remédios que são prescritos pelos médicos quando eu } \\
\text { possuo dores, enxaqueca, alergia, picos febris e rinite com frequência, sendo inviável ir ao } \\
\text { médico todas às vezes. Estava com determinados sintomas e por já ter realizada consulta médica } \\
\text { anteriormente para os mesmos sintomas, resolvi utilizá-los novamente. }\end{array}$ \\
\hline
\end{tabular}

\section{DISCUSSÃO}

Este trabalho caracterizou a prática de automedicação entre universitários do curso de Enfermagem. Nossos resultados mostraram que 86,5\% dos acadêmicos da primeira série e $100 \%$ da quarta série realizam essa prática, dados esses que mostram existir associação estatisticamente significativa entre a prática de se automedicar e a série que o estudante está matriculado. Esses resultados se assemelham ao encontrado na literatura ${ }^{(15)}$.

Pensando neste grave problema de saúde pública e atendendo a uma recomendação da OMS, o Ministério da Saúde criou o Comitê Nacional para a Promoção do Uso Racional de Medicamentos por meio da Portaria GM no $1.956 / 06^{(16)}$. Seu papel é o desenvolvimento de ações estratégicas para ampliar o acesso da população à assistência farmacêutica e para melhorar a qualidade e a segurança na utilização dos medicamentos.

Dentre os medicamentos utilizados na forma de automedicação, os analgésicos apresentaram as maiores utilizações indiscriminadas por serem usados no alívio da dor ${ }^{(11,13)}$, por possuírem fácil aquisição e em grande parte por apresentarem venda livre, não necessitando de receita médica ${ }^{(17)}$.

O hábito de armazenar medicamentos no domicílio, na maioria das vezes por não ter utilizado a quantidade prescrita ou por ter adquirido uma quantidade maior, pode justificar o aparecimento de antibióticos na relação dos medicamentos utilizados para automedicação. Nesse sentido, vale ressaltar que esta pesquisa foi realizada após a Resolução da Diretoria Colegiada - RDC 44/2009, que dispõe sobre o controle de medicamentos à base de substâncias classificadas como antimicrobianos, de uso sob prescrição médica, isolada ou em associação(18).

Observou-se associação estatisticamente significativa entre a prática de se automedicar com antiinflamatórios e as séries, mesmo após os estudantes da quarta série terem cursado a disciplina de 
Farmacologia. Esperava-se que o conhecimento farmacológico das reações adversas ao uso dessa medicação pudesse influenciar nessa prática, fato este não corroborado neste estudo. Demonstra-se que o acúmulo de conhecimento, seja ele adquirido nas instituições educacionais ou em experiências de vida, gera uma maior confiança naqueles que se automedicam $^{(1)}$. Atualmente, é possível encontrar, disponível na internet, as bulas de vários medicamentos. Essa forma de acesso ao conhecimento difunde as informações do fármaco, bem como suas indicações. Porém, as bulas dos medicamentos ainda são incompletas, não apresentando de maneira clara alguns riscos do uso do medicamento ou mesmo todas as suas interações, além do fato de que a indústria farmacêutica demora em atualizar eventos adversos encontrados nos estudos baseados em evidências. A leitura da bula do(s) medicamento(s) em uso, como única fonte de informação, foi prática ativa entre os estudantes de ambas as séries, sendo maior na quarta série, prática essa presente também entre acadêmicos do curso de medicina $^{(1)}$.

No presente estudo, observa-se que grande parte dos estudantes não relatou a ocorrência de efeitos adversos durante o uso dos medicamentos, mesmo sendo os que possuem maior conhecimento relativo a esses efeitos ${ }^{(19)}$. Apesar do número superior de estudantes que confirmam a prática de automedicação na quarta série, observa-se que os recém-ingressados referem mais a ocorrência de efeitos adversos, efeitos esses que podem ser mais bem identificados após a orientação de um profissional médico ${ }^{(20)}$. Esses efeitos correspondem a $5 \%$ da entrada em hospitais, afeta de 6 a $7 \%$ dos pacientes já hospitalizados, e contribui para a morte em até $32 \%$ deles $^{(21)}$.

Estudo realizado para a Associação Nacional de Farmácias (ANF) mostra que os riscos da automedicação são acrescidos conforme aumenta o número de medicamentos que o indivíduo ingere concomitantemente, prática essa conhecida como polifarmácia, e presente entre os estudantes da primeira série $(62,1 \%)$ e da quarta série $(78,1 \%)$. Estão implicados na gênese da polifarmácia aspectos como o número de médicos consultados, a ausência de perguntas durante a consulta médica sobre as medicações em uso e a automedicação, além da presença de comorbidades ${ }^{(22)}$.

Em relação aos dados qualitativos, apresentados no Quadro 1, observa-se que a ideia central "pela indicação de terceiros", mostra um sujeito coletivo que confia na eficácia dos medicamentos utilizados anteriormente por familiares, amigos, balconistas de farmácia, etc. Estudo que avaliou as características de automedicação entre estudantes de enfermagem mostrou que 33,9\% dos participantes fizeram automedicação por indicação de alguém da família(13). O hábito de recorrer a alguém próximo, não médico, mas com algum conhecimento empírico, para aconselhamento sobre o que utilizar para o tratamento de algum mal que o aflija, faz parte da ontologia da saúde do nosso povo. Essa cultura é perpetuada de geração em geração, de pais para filhos, principalmente porque o acesso ao atendimento médico nesse país continua limitado, apesar do acesso à saúde ter progredido do Brasil-Colônia para o modelo universal e integral da saúde na atual Constituição. Dados da literatura mostram que, entre universitários de diferentes cursos da área da saúde, as porcentagens de automedicação por indicação de terceiros variam de $54,8 \%$ na enfermagem, 57,4\% na farmácia, 60,2\% na odontologia a $70,6 \%$ na medicina, normalmente realizadas por pessoas não qualificadas ${ }^{(11)}$. Em Portugal, $50 \%$ das prescrições são feitas por farmacêuticos ${ }^{(23)}$. Esse profissional faz as prescrições de medicamentos de venda livre, denominados "Over-the-Counter" (OTC) e também assume a importante missão de promover a automedicação responsável, ou seja, a automedicação sob indicação farmacêutica, promovendo assim o uso racional do medicamento(24). Neste sentido, a partir de 2013, o Conselho Regional de Farmácia, órgão que regulamenta a profissão farmacêutica no Brasil, instituiu a prescrição dos medicamentos de venda livre (OTC), pelo profissional farmacêutico, com o propósito de reduzir a automedicação no país e atender a Política Nacional de Medicamentos, que propõe o uso racional de medicamentos.

A ideia central "pela falta de tempo" mostra um sujeito coletivo que refere ser este o principal motivo para a prática de automedicação. Neste sentido, deve-se considerar que o currículo no qual os estudantes estão inseridos é orientado por competências e as atividades são organizadas em tempo integral, propiciando pouca disponibilidade de tempo livre, o que poderia justificar essa prática. Estudos sobre a automedicação em acadêmicos da área da saúde mostram que 18,6\% dos entrevistados relataram não possuir tempo para ir a uma consulta médica ${ }^{(10)}$. Porcentagens maiores $(34,8 \%$ e $50 \%$ ) também foram observadas ${ }^{(1,13)}$.

A ideia central "para economizar tempo", formulada a partir das respostas dadas pelos estudos antes da quarta série, mostra um sujeito coletivo que prefere 
automedicar-se, em detrimento da procura de um atendimento médico, por afirmar que "perde tempo ao procurar e ao esperar por uma consulta médica". Devese considerar que parte dos entrevistados da quarta série, após o estudo da disciplina de Farmacologia, considera deter conhecimento suficiente para a prática de automedicação, além de obterem informações através da bula da medicação.

A ideia central "ter conhecimento dos efeitos dos medicamentos" mostra um sujeito coletivo que afirma possuir conhecimento sobre a ação $\mathrm{da}(\mathrm{s})$ medicação(ões) em uso, independente da forma de aquisição deste conhecimento (leitura da bula, internet, livros, etc). Esses resultados se assemelham aos observados com estudantes do curso de medicina(1). Na quarta série, foi identificada uma ideia central semelhante, intitulada de "possuir conhecimentos científicos". A aquisição de conhecimentos científicos sobre medicamentos durante a graduação ocorre, principalmente, quando os estudantes cursam a disciplina de Farmacologia(11). Os conteúdos dessa disciplina, na Faculdade de Medicina de Marília, estão distribuídos ao longo das duas séries iniciais do curso de Enfermagem e em situações reais vivenciadas na prática (terceira e quarta séries). Sendo assim, acredita-se que os conhecimentos relatados pelos estudantes da quarta série, no presente trabalho, estejam relacionados aos adquiridos ao longo de sua formação.

A ideia central "ter o mesmo problema", formulada a partir das respostas dadas pelos estudantes da primeira série, mostra um sujeito coletivo que conhece o quadro clínico que apresenta, pois em outra ocasião foi consultar o médico e este prescreveu medicações que surtiram efeito, sendo então reutilizadas. Na quarta série, foi identificada uma ideia central semelhante "utilizar diagnósticos prévios", em que é possível observar que o sujeito coletivo reutiliza medicações prescritas anteriormente, afirmando apresentar sinais/sintomas recorrentes. Comparando a prática de automedicação entre estudantes universitários paquistaneses, com idade média de 21 anos, observa-se que um dos principais motivos que levaram à automedicação foi conhecer os sintomas, o que auxiliou na escolha do medicamento ${ }^{(25)}$.

A ideia central "para o alívio da dor", formulada a partir das respostas dadas pelos estudantes da primeira série, mostra um sujeito coletivo que afirma ser este um sintoma normal e de fácil resolução, não necessitando ir novamente ao médico, para que este prescreva a mesma medicação. Ao se pesquisar dor crônica e automedicação entre estudantes de diferentes séries do curso de
Enfermagem, sugere-se que 69,7\% automedicaram-se para o alívio deste sintoma ${ }^{(13)}$. Dados da literatura mostram que a dipirona é o analgésico mais utilizado $(59,8 \%)$ e proporciona alívio da dor classificado como bom ${ }^{(13)}$, sugerindo que esse alívio pode postergar o esclarecimento do diagnóstico bem como o tratamento adequado da dor.

A ideia central "pela demora no agendamento" formulada a partir das respostas dadas pelos estudantes da primeira série mostra um sujeito coletivo que pratica automedicação por não conseguir acesso ao sistema de saúde, possivelmente devido à demanda elevada e também pelo reduzido número de profissionais de saúde, dados esses corroborados por pesquisa Datafolha, em 2014, que verificou que a falta de profissional médico é um dos maiores problemas do Sistema Único de Saúde SUS. Estudantes de medicina da mesma instituição relatam que a dificuldade de acesso aos serviços de saúde colabora para a prática de automedicação ${ }^{(1)}$.

A ideia central "pela facilidade de acesso ao medicamento", formulada a partir das respostas dadas pelos estudantes da quarta série, mostra um sujeito coletivo que pratica automedicação pelo fácil acesso à medicações. Essa prática pode estar apoiada pela venda livre da maioria dos medicamentos citados como utilizados na automedicação, ou pelo armazenamento dos mesmos nas residências dos usuários ${ }^{(17)}$.

\section{CONCLUSÃO}

Frente ao exposto, observa-se que a automedicação é uma prática frequente entre os estudantes do curso de Enfermagem, assim como em universitários de outros cursos e de outras instituições de ensino superior. Esta prática também está presente em outros segmentos da população.

Dentre os medicamentos que predominam nessa prática, observa-se a presença de analgésicos e antiinflamatórios, possivelmente pelo fácil acesso aos mesmos, acesso esse apontado nas ideias centrais e nos discursos do sujeito coletivo, os quais expressam características que podem explicar a prática de automedicação, mesmo tendo eles alto potencial de efeitos adversos.

A universidade é vista como uma fonte geradora de grandes conhecimentos para os estudantes da área da saúde, mas, conhecimentos esses que não foram significativos para redução do comportamento de automedicação, ao contrário, propiciam aos estudantes a falsa ideia que estão mais aptos para essa prática. Neste sentido, explicita-se a necessidade de novas reflexões 
sobre a abordagem da temática automedicação no conteúdo programático de Farmacologia, fazendo analogia com o uso racional de medicamentos segundo as políticas governamentais atuais.

\section{REFERÊNCIAS}

1. Masson W, Furtado PL, Lazarini CA, Conterno LO. Automedicação entre acadêmicos do curso de medicina da Faculdade de Medicina de Marília, São Paulo. Rev Bras Pesq Saúde. 2012 [acesso em: 11 de fev. 2014];14(4):82-89. Disponível em:

http://www.periodicos.ufes.br/RBPS/article/view/5123/3849. 2.Silva JAC, Gomes AZ, Oliveira JPS, Sasaki YA, Maia BTB, Abreu BM. Prevalência de automedicação e os fatores associados entre os usuários de um Centro de Saúde Universitário. Rev Bras Clin Med. 2013 [acesso em: 11 de fev. 2014];11(1):27-30. Disponível em: http://files.bvs.br/upload/S/16791010/2013/v11n1/a3385.pdf.

3.Schuelter-Trevisol F, Trevisol DJ, Jung GS, Jacobowski B. Automedicação em universitários Rev Bras Clin Med. 2011 [acesso em: 11 de fev. 2014];9(6):414-7. Disponível em: http://files.bvs.br/upload/S/1679-1010/2011/v9n6/a2556. 4. Becker, CB. Os escravos e sua relação com a história da saúde na fronteira meridional do Rio Grande do Sul do século XIX. X Encontro Estadual de História. O BRASIL NO SUL. 26 a 30 de julho de 2010. Santa Maria - RS, Universidade Federal de Santa Maria. Disponível em:

http://www.eeh2010.anpuhrs.org.br/resources/anais/9/1278786 544_ARQUIVO_artigonpuhoriginal.pdf.

5.Chaves GC, Emmerick I, Pouvourville N, Saint-Denis T, Fonseca ASA, Luiza VL. Indicadores de uso racional de medicamentos e acesso a medicamentos: um estudo de caso. Rev Bras Farmacologia. 2005 [acesso em: 11 de fev. 2014]; 86(3):97103. Disponível em:

http://revbrasfarm.org.br/pdf/2005/V86_N3_2005/pag_97a103_ INDICADORES.pdf.

6.Casagrande EF, Almeida AC, Mello NRS, Oliveira RN, Oliveira TB, Pereira VNM et al. Estudo da utilização de medicamentos pela população universitária do município de Vassouras (RJ). Infarma. 2004,16(5/6):86-88.

7. Agencia Nacional De Vigilância Sanitária. - Protocolo de segurança na prescrição, uso e administração de medicamentos. Ministério da Saúde. Protocolo coordenado pelo Ministério da Saúde e ANVISA em parceria com FIOCRUZ e FHEMIG. Disponível em:

http://www.anvisa.gov.br/hotsite/segurancadopaciente/documen tos/julho/Protocolo\%20Identifica\%C3\%A7\%C3\%A30\%20do\%20 Paciente.pdf. [Acesso em: 03 de abr. 2014].

8. João WSJ. Reflexões sobre o Uso Racional de Medicamentos. Pharmacia Brasileira. 2010 [acesso em: 11 de fev. 2014];(78). Disponível em:

http://www.cff.org.br/sistemas/geral/revista/pdf/128/015a016_a rtigo_dr_walter.pdf.

9. Silva JR, Souza M, Paiva AS. Avaliação do uso racional de medicamentos e estoque domiciliar. Ensaios e Ciência: Ciências Biológicas, Agrárias e da Saúde. 2012;16(1): 109-124.

10. Aquino DS, Barros JAC, Silva, MDP. A automedicação e os acadêmicos da área de saúde. Ciências e Saúde Coletiva. 2010 [acesso em: 11 de fev. 2014];15(5):2533-38. Disponível em http://www.scielosp.org/pdf/csc/v15n5/v15n5a27.pdf. 11. Silva LSF, Costa AMDD, Terra FS, Zanetti HHV, Costa RD, Costa MD. Automedicação em acadêmicos de cursos de graduação da área da saúde de uma universidade privada do Sul do Estado de Minas Gerais. Odontologia Clínica-Científica. 2011 [acesso em: 11 de fev. 2014]; 10(1):57-63. Disponível em: http://revodonto.bvsalud.org/pdf/occ/v10n1/a11v10n1.pdf. 12. Galato D, Madalena J, Pereira GB. Automedicação em estudantes universitários: a influência da área de formação. Ciência Saúde Coletiva. 2012 [acesso em: 11 de fev. 2014];17(12):3323-30. Disponível em: http://www.scielosp.org/pdf/csc/v17n12/17.pdf. 13.Souza LAF, Silva D, Ferraz GC, Sousa FAEF, Pereira LV. Prevalência e caracterização da prática de automedicação para alívio da dor entre estudantes universitários de enfermagem. Rev Latino-Am Enf. 2011 [acesso em: 11 de fev. 2014];19(2):[7 telas]. Disponível em: http://www.scielo.br/pdf/rlae/v19n2/pt_04.pdf.

14. Lefèvre $F$, Lefèvre $A M C$. Os novos instrumentos no contexto da pesquisa qualitativa. In: Lefèvre, F., Lefèvre, A. M. C.; Teixeira, J. J. V.; organizadores. O discurso do sujeito coletivo: uma nova abordagem metodológica em pesquisa qualitativa. Caxias do Sul: EDUCS, p.11-35, 2000.

15. Damasceno DD, Terra FS, Zanetti HHV, DïAndrá ED, Silva HLR, Leite JA. Automedicação entre graduandos de enfermagem, farmácia e odontologia da Universidade Federal de Alfenas. Rev Min Enf. 2007 [acesso em: 11 de fev. 2014]; 11(1):48-52. Disponível em http://www.reme.org.br/artigo/detalhes/312. 16. Ministério da Saúde. Plano de Ação: comitê nacional para promoção do uso racional de medicamentos. Disponível em: http://portal.saude.gov.br/portal/arquivos/pdf/portaria_1956_23 _ago_2006.pdf 17. Tierling VL, Paulinoa MA, Fernandesa LC, Schenkelb EP, Mengueet SS. Nível de conhecimento sobre a composição de analgésicos com ácido acetilsalicílico. Rev Saúde Pública. 2004 [acesso em: 05 de set. 2011],38(2):223-227. Disponível em: http://scielo.br/pdf/rsp/v38n2/19782.pdf.

18. Agencia Nacional De Vigilância Sanitária. Resolução da Diretoria Colegiada - RDC no 44, de 17 de agosto de 2009 [acesso em: 11 de fev. 2014]. Disponível em http://www.anvisa.gov.br/divulga/noticias/2009/pdf/180809_rdc 44.pdf.

19.Ribeiro MI, Oliveira A, Silva H, Mendes M, Almeida M, Silva T. Prevalência da automedicação na população estudantil do Instituto Politécnico de Bragança. Rev Port Saude Pub [Internet]. 2010 [acesso em: 11 de fev. 2014]; 28(1):41-48. Disponível em:

http://www.scielo.gpeari.mctes.pt/scielo.php?script=sci_arttext\& pid=S0870-90252010000100005\&Ing=pt.

20. Marchi KC, Bárbaro AM, Miasso AI, Tirapelli CR. Ansiedade e consumo de ansiolíticos entre estudantes de enfermagem de uma universidade pública. Rev Eletr Enf. [Internet]. 2013 [acesso em: 11 de fev. 2014]; 15(3):731-9. Disponível em:

http://dx.doi.org/10.5216/ree.v15i3.18924.

21. Malbran A, Yeyati E, Rey GL. Diclofenac induces basophil degranulation without increasing CD63 expression in sensitive patients. Journal Translational Immunology. 2007,147(1):99105. Disponível em:

http://www.ncbi.nlm.nih.gov/pmc/articles/PMC1810452/.

22. Rollason $V$, Vogt N. Reduction of polypharmacy in the elderly: a systematic review of the role of the pharmacist. Drugs and Aging. 2003,20(11):817-832.

23. Mendez Z, Martins AP, Miranda AC, Soares MA, Ferreira AP, Nogueira A. Prevalência da automediação na população urbana portuguesa. Rev Bras Cien Farmaceuticas. 2004,40(1):21-5. 24. Joaquim MR. Automedicação versus indicação farmacêutica. Dissertação de mestrado. Ciências Farmacêuticas, Faculdade de Ciências e Tecnologia, Univ. do Algarve, 2011 [acesso em: 11 de fev. 2014]. Disponível em: http://hdl.handle.net/10400.1/1746. 25. Zafar SN, Syed R, Sana W, Zubairi AJ, Vaqar T, Shaikh M, et al. Self-medication amongst University Students of Karachi: Prevalence, Knowledge and Attitudes. J Pakistan Med Assoc. 2008,58(4):214-7.

Artigo recebido em 29/10/2012.

Aprovado para publicação em 07/02/2014. Artigo publicado em 30/09/2014. 